P.N. Vuong ${ }^{1}$

S. Wanji ${ }^{2}$

J. Prod'Hon ${ }^{3}$

O. Bain ${ }^{2}$

\title{
Nodules sous-cutanés et lésions cutanées engendrés par diverses onchocerques chez des bovins africains*
}

VUONG (P.N.), WANJI (S.), PROD'HON (J.), BAIN (O.). Nudules sous-cutanés et lésions cutanées engendrés par diverses onchocerques chez des bovins africains. Revue Élev. Méd. vét. Pays trop., 1994, 47 (1): $47-51$

Des nodules sous-cutanés et de la peau ombilicale sont prélevés sur 6 Bos indicus onchocerquiens au Cameroun, pour l'étude anatomopathologique. Les nodules à Onchocerca ochengi et à Onchocerca dukei ont la même structure que celle des nodules à Onchocerca volvulus de l'homme ; ce sont des pseudo-kystes inflammatoires contenant souvent une filaire femelle. La composante cellulaire de la paroi du pseudo-kyste permet de classer les nodules en 3 types : nodule "jeune", nodule "évolué" et nodule "ancien". Le nodule est circonscrit par des vaisseaux dont la lumière contient parfois des microfilaires et des morulas. La peau, parasitée par les microfilaires de ces deux onchocerques et par celles de $O$, gutturosa et $O$. armillata, présente diverses lésions de dermite avec sclérose cicatricielle, superposables à celles observées chez les malades onchocerquiens. Dans la majorité des cas, les infilltrats inflammatoires circonscrivent les capillaires lymphatiques longeant les vaisseaux sanguins réalisant une lymphangite.Toutes ces lésions sont superposables à celles observées chez les malades onchocerquiens. La présence de microfilaires au sein du tissu de granulation bordant le pseudo-kyste montre que ces microfilaires sont pondues dans la lumière du pseudo-kyste. Seules celles qui échappent à la réaction inflammatoire arrivent à pénétrer dans les lymphatiques de la capsule du nodule. Elles peuvent alors emprunter le système lymphatique pour arriver dans le derme superficiel. Les onchocerques nodulaires bovines constituent un modèle intéressant pour l'onchocercose humaine.

Mots-clés : Bovin - Onchocercose - Lésion- Maladie de la peau - Maladie du système lymphatique - Anatomopathologie - Cameroun.

\section{INTRODUCTION}

Les bovins africains sont fréquemment parasités par quatre espèces d'onchocerques : $O$. gutturosa Neumann,1910, logée au niveau des ligaments cervicaux, $O$. armillata Railliet et Henry, 1908, située dans l'intima de l'aorte, et deux espèces nodulaires, $O$. ochengi Bwangamoi, 1969, intradermique et sous-cutanée, et $O$. dukei Bain, Bussiéras et Amégée, 1974, plus profonde, dans les muscles striés. Ces deux dernières espèces appar-

1. Unité d'anatomie et de cytologie pathologiques, Hôpital SaintMichel, 33 rue Olivier de Serres, 75015 Paris, France.

2. Laboratoire de biologie parasitaire, protistologie et helminthologie, CNRS-URA 114, Muséum national d'histoire naturelle, 61 rue Buffon, 75231 Paris Cedex 05, France.

3. ORSTOM, Institut Pasteur Yaoundé, BP 1274, Yaoundé, Cameroun.

Reçu le 22.4.1993, accepté le 9.2.1994.

* Etude subventionnée par UNDP/World Bank/WHO, Special Programme for Research and Training in Tropical Diseases (TDR $\mathrm{N}^{\circ}$ T16/181/05/56) et par la CEE (TS2-M-0067) tiennent à la même lignée que 0 . volvulus (1) et leurs nodules sont de plus petite taille que ceux du parasite humain. Ce matériel, qui n'a pas encore fait l'objet de recherches particulières, nous a donc paru propice pour essayer de comprendre, par une analyse histo-pathologique, l'origine et la genèse des nodules d'onchocerques et voir quels sont leurs liens avec le système lymphatique. En même temps, les prélèvements cutanés associés aux nodules nous ont permis d'obtenir de nouveaux documents concernant la localisation tissulaire des microfilaires d'onchocerques et la nature des lésions induites.

\section{MATÉRIEL ET MÉTHODE D'ÉTUDE}

Le matériel a été récolté à Touboro, au Cameroun, lors du contrôle sanitaire effectué à l'abattoir. II comprend des prélèvements cutanés et 8 nodules sous-cutanés récoltés dans la région ombilicale de 6 Bos indicus. Les nodules parasités par $O$. ochengi proviennent de 5 bovins, ceux parasités par $O$. dukei sont récoltés chez un seul animal. Lors de la récolte du matériel, des "snips" cutanés ombilicaux ont été dilacérés dans le sérum physiologique pour rechercher les microfilaires. L'examen direct de ces microfilaires permet d'identifier l'espèce des onchocerques (2). Les infections sont mixtes dans tous les cas : $O$. gutturosa et $O$. ochengi sont constamment présents, $O$. dukei l'est chez deux animaux, ces trois premières espèces ayant des densités microfilariennes souvent très fortes ; 0 . armillata est détecté une fois, alors qu'on sait que 100 p. 100 des animaux ont ce parasite, mais ses microfilaires sont toujours en faible densité. Les prélèvements destinés à l'étude histo-pathologique sont préparés selon la méthode habituelle (24).

\section{RÉSULTATS}

\section{Filaires adultes, microfilaires et nodules}

Les nodules à $O$. ochengi et à $O$. dukei sont de même type et ne se distinguent que par leur situation tissulaire, plus ou moins profonde. Les descriptions qui sont faites ci-dessous concernent indifféremment ces deux espèces. Les nodules constituent des pseudo-kystes (c'est-à-dire des cavités bordées par un tissu inflammatoire) dont la lumière est souvent occupée par une filaire femelle. 
P.N. Vuong S. Wanji J. Prod'Hon O. Bain

Dans tous les cas, la filaire est entourée d'une couche de matériel fibrineux d'épaisseur inégale, éosinophile à l'hématéine-éosine-safran. Le parasite est circonscrit par un tissu inflammatoire dont la composante cellulaire, disposée en strates de l'intérieur vers l'extérieur, permet de classer les nodules en 3 types :

- nodule "jeune" : la filaire est entourée par une réaction inflammatoire qui, de l'intérieur vers l'extérieur, est aiguë, ensuite sub-aiguë et granulomateuse, pourvue de granulomes à cellules géantes multinucléées (fig. 1 et 2). Sur certaines sections, les granulomes à corps étranger s'organisent autour des débris détachés de la couche de matériel fibrineux enveloppant le parasite ;

- nodule "évolué" : il comporte essentiellement des lésions inflammatoires subaiguës et granulomateuses (fig. 3 et 4 ) ;

- nodule "ancien" : il est caractérisé par la détersion quasi-totale du tissu inflammatoire qui est remplacé par un tissu scléro-hyalin enserrant des fragments de filaire altérée.

Le nodule est limité par des travées scléreuses réalisant une capsule conjonctive plus ou moins épaisse. Cette capsule conjonctive contient des capillaires sanguins néoformés se prolongeant de façon radiaire dans le tissu inflammatoire (fig.5). Celui-ci renferme quelques microfilaires plus ou moins bien conservées. Des capillaires lymphatiques néoformés ont été également identifiés à deux reprises. Un stade embryonnaire d'Onchocerca spp. (une morula) a été trouvé dans la lumière d'un de ces capillaires lymphatiques et, dans un autre cas, une microfilaire (fig.6).

La classification des nodules est en fait schématique puisque, dans un nodule "ancien", une partie de la filaire est entourée par des lésions inflammatoires sub-aiguës et granulomateuses à corps étranger.

\section{Microfilaires extra-nodulaires et lésions cutanées}

Des sections de microfilaires ont été trouvées à la fois dans le tissu conjonctif du derme et dans la lumière des capillaires lymphatiques (fig. 7 et 8 ). Ces structures Iongent les capillaires sanguins, reconnaissables par la présence des hématies. Dans un cas, un vaisseau lymphatique contient un fragment de morula (fig. 9). Le tissu conjonctif présente diverses lésions inflammatoires de répartition et d'intensité variables :

- dermite chronique non spécifique avec infiltration de cellules mononucléées lympho-histiocytaires ;

- dermite aiguë essentiellement vasculo-exsudative avec diapédèse des hématies et des polynucléaires ;

- dermite sub-aiguë avec infiltration par endroit nodulaire, ailleurs diffuse, de cellules inflammatoires mononucléées : lymphocytes, plasmocytes, histiocytes. Dans la

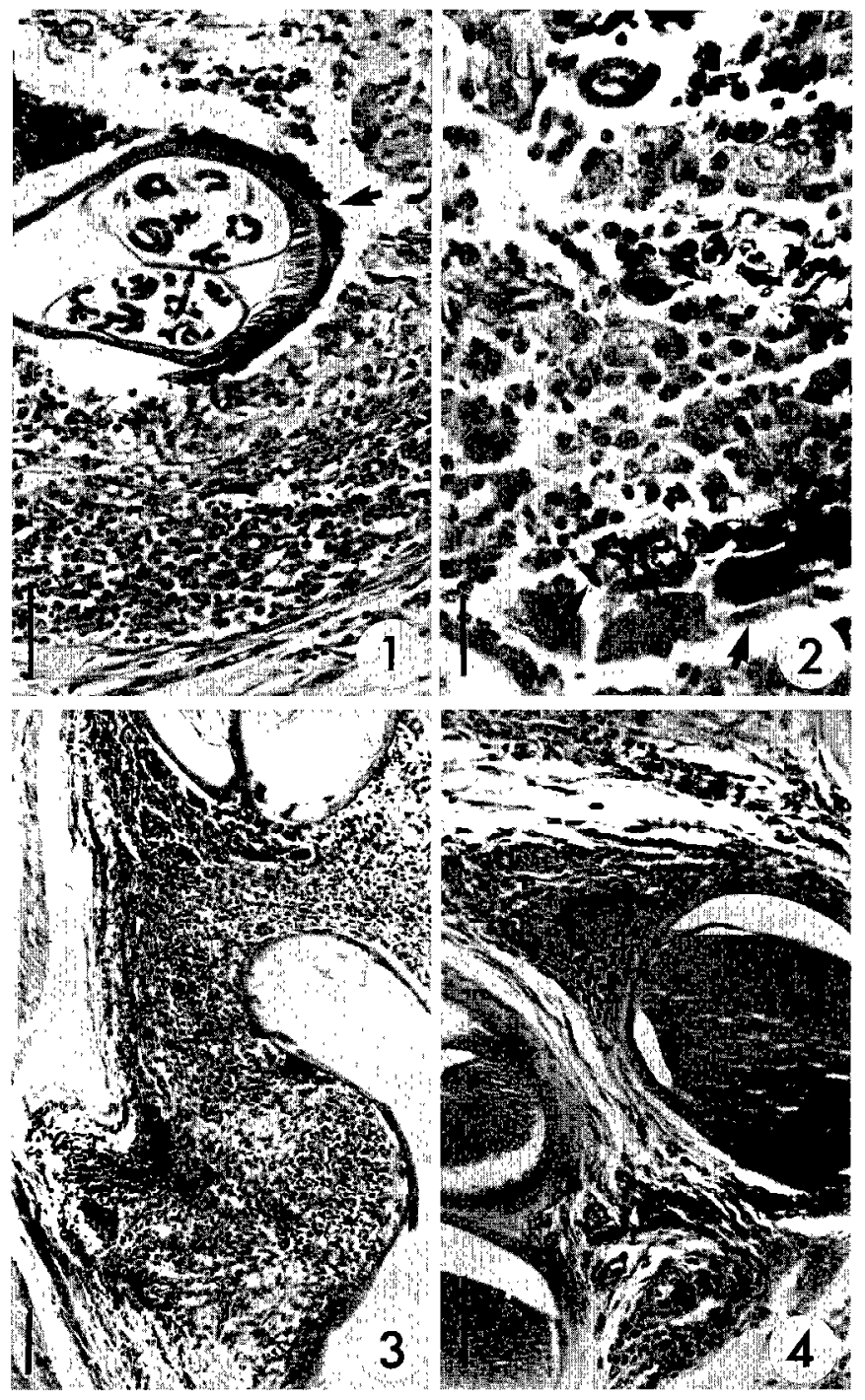

Figure 1: O. dukei chez Bos indicus. Vue d'ensemble d'un nodule onchocerquien "jeune". La lumière du nodule est occupée par une filaire femelle dont l'utérus contient des microfilaires. La cuticule du parasite est épaissic par une ganguc protéique réalisant le phénomène de SplendoreHoeppli (flèche). En périphérie, le parasite est entouré par une réaction inflammatoire qui, de l'intérieur vers l'extérieur, est aiguë, ensuite subaiguë et granulomateuse pourvue de granulomes à cellules géantes multinucléées.

Figures 2 à 4 : nodules à $\mathrm{O}$. ochengi chez Bos indicus. 2 : sur certaines sections, les granulomes à corps étranger (flèche) s'organisent autour des débris détachés de la couche de matériel fibrineux enveloppant le parasite. 3 4 : nodule "évolué": il comporte essentiellement des lésions inflammatoires sub-aiguës (3) et granulomateuses (4) (Echelle, une barre $=100 \mu \mathrm{m}$ ). 

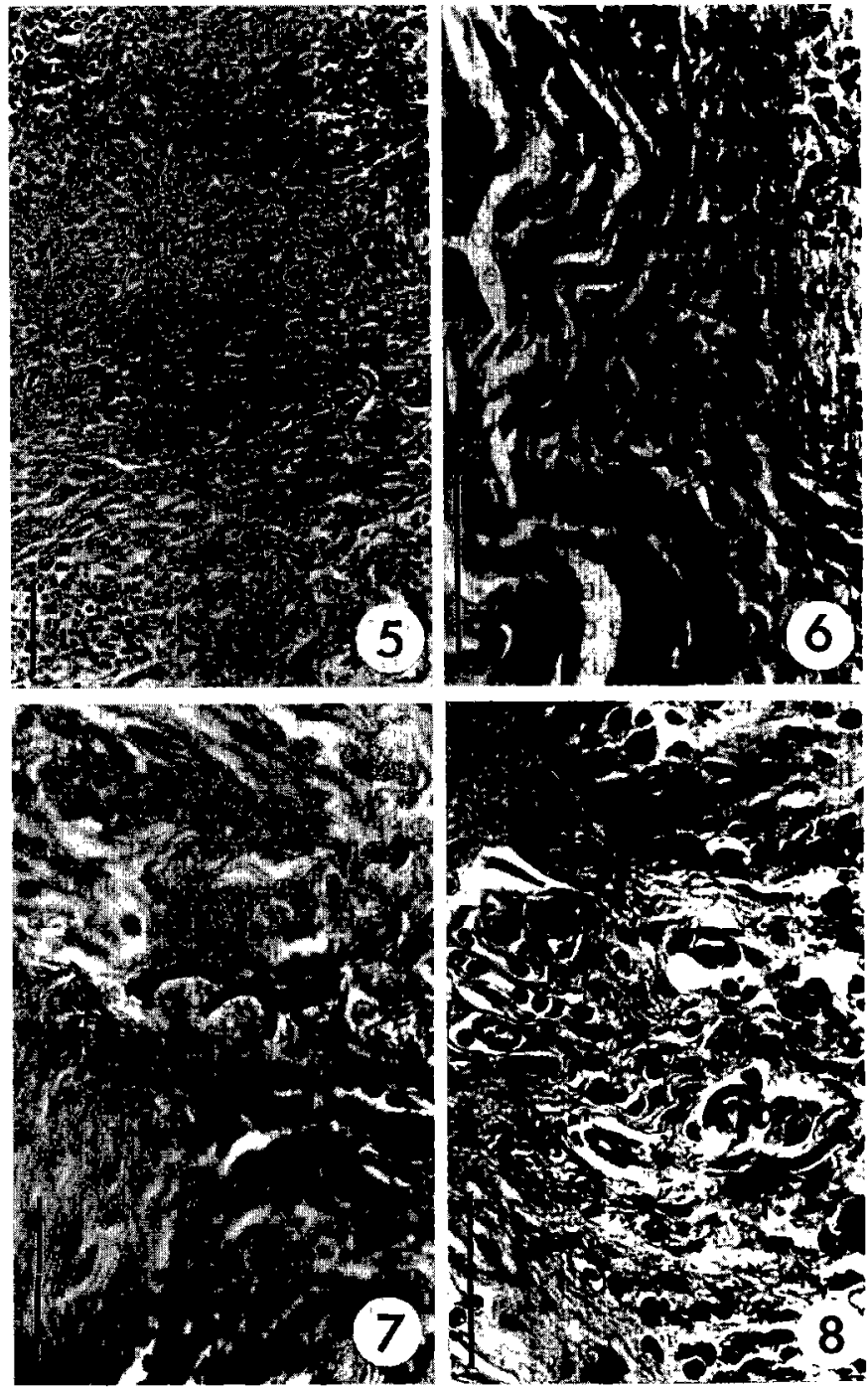

Figures 5 et 6 : nodules à $\mathrm{O}$. ochengi chez Bos indicus. $5:$ la capsule conjonctive contient des capillaires sanguins néoformés se prolongeant de façon radiaire dans le tissu inflammatoire qui comporte des microfilaires (flèche) plus ou moins bien conservées. 6 : une microfilaire (flèche) a été retrouvée dans la lumière d'un capillaire lymphatique longeant la capsule. $($ Echelle, une barre $=100 \mu \mathrm{m})$.

Figures 7 et 8 : peau de Bos indicus parasitée par des microfilaires d'Onchocerca spp. Des sections de microfilaires ont été retrouvées à la fois dans le tissu conjonctif du derme et dans la lumière des capillaires lymphatiques. Ces structures longent les capillaires sanguins (Echelle, une barre $=50 \mu \mathrm{m}$ )

majorité des cas, ces infiltrats inflammatoires circonscrivent les capillaires lymphatiques longeant les capillaires sanguins et les arteres musculaires de petit et de moyen calibres ;



Figures 9 et 10 : peau de Bos indicus parasitée par des microfilaires d'Onchocerca spp. 9 : dans un cas, un vaisseau lymphatique contient un fragment de morula (flèche). 10 : dermite granulomateuse avec formation de granulomes à corps étrangers. (Echelle, une barre $=50 \mu \mathrm{m}$ ).

- dermite granulomateuse avec formation de granulomes à corps étrangers (fig.10) ;

- sclérose cicatricielle contenant des capillaires sanguins et/ou lymphatiques néoformés et quelques cellules mononucléées.

Aucune lésion n'est observée dans la paroi des artères musculaires du derme profond. Certaines lésions réactionnelles d'intensité variable sont notées : ectasie des vaisseaux lymphatiques et des capillaires sanguins, infiltration de mastocytes, infiltration de mélanophages,...

\section{DISCUSSION}

\section{Les nodules à onchocerques}

Bien que les nodules à $O$. ochengi et $O$. dukei n'aient pas la même localisation - les premiers sont dans l'aponévrose sous-cutanée, les seconds dans le muscle strié -, leur aspect anatomo-pathologique apparaît similaire. Cet aspect est superposable à celui des nodules à $O$. volvulus de l'homme, décrit de nombreuses fois $(5,6,11,14,16)$. Les nodules à $O$. gibsoni Cleland et Johnston, 1910 (8) des bovins asiatiques et australiens présentent aussi la même structure (on cite la première étude faite par GILRUTH et SWEET, 1911) (10). 
Quelques remarques peuvent être faites au sujet de ces nodules :

- L'existence de polynucléaires plus ou moins altérés au contact des filaires adultes, réalisant une lésion inflammatoire aiguë, est vraisemblablement liée à un processus traumatique dû à la mobilité des vers à l'intérieur du nodule.

- Le polymorphisme des lésions inflammatoires au niveau des nodules dits "anciens" peut indiquer deux éventualités, qui ne s'excluent pas : $1^{\circ}$ ) la mort de la filaire est progressive, atteignant segment par segment, ce qui correspond aux observations morphologiques faites sur les vers extraits des nodules $\left.(17,19) ; 2^{\circ}\right)$ le nodule peut contenir plusieurs filaires enchevêtrées comme l'ont montré SCHULZ-KEY et ALBIEZ pour O. volvulus en 1977 (20), ou BEVERIDGE et al. en 1980 pour O. gibsoni (3), qui sont différemment altérées.

- L'intensité du processus inflammatoire ne semble pas lié à la mort de la filaire, contrairement à ce qui a été indiqué pour $O$. armillata (7) ou $O$. cervicalis Railliet et Henry, 1910 (15). Quant à la sclérose donnant naissance à la capsule du nodule, elle s'intègre, comme on l'a confirmé, dans l'évolution habituelle du processus inflammatoire et n'est pas liée à la constitution anatomique du ver, en particulier à sa cuticule.

- Le matériel fibrineux qui entoure les filaires, vraisemblablement protéique, constitue le phénomène de Splendore-Hoeppli (12), dont la signification pathogénique est discutable. Ce phénomène, évoqué par CONNOR et NEAFIE (9) chez les onchocerquiens, pourrait correspondre soit à un "camouflage" des filaires adultes qui s'habillent d'une couche protéique provenant de la concrétion de l'insudation plasmatique de l'hôte dans la lumière du pseudo-kyste ; soit il pourrait traduire une réaction immunologique, avec accumulation des immunoglobulines de l'hôte autour des parasites, tendant ainsi à les immobiliser.

- Il n'a pas été possible d'identifier l'origine de la capsule conjonctive circonscrivant le nodule filarien. Une hypothèse parfois suggérée, par exemple par BRUMPT (4), est que les nodules se formeraient à partir de vaisseaux lymphatiques parasités par des filaires et profondément transformés sous l'effet de la réaction inflammatoire. Une telle hypothèse semble en effet s'accorder avec les quelques cas d'onchocerques libres dans des vaisseaux ou des ganglions lymphatiques, observés soit chez l'homme $(13,18)$, soit chez les bovins (8). Dans les nodules étudiés ici, il n'a pas été retrouvé de fibres musculaires ou de structures élastiques permettant de démontrer la nature lymphatique de la capsule. Des vaisseaux lymphatiques ont été trouvés dans le tissu inflammatoire et la capsule conjonctive circonscrivant le nodule ; ils longent les capillaires sanguins et, comme eux, traversent la capsule et ont une disposition radiaire dans le tissu inflammatoire.

\section{Microfilaires intra et extra-nodulaires}

La présence de microfilaires au sein du tissu de granulation bordant le pseudo-kyste montre que ces microfilaires sont pondues dans la lumière du pseudo-kyste. Elles traversent le tissu de granulation ; la plupart sont détruites par la réaction inflammatoire. Seules les microfilaires (ou les morula exceptionnellement pondues) qui échappent à la réaction inflammatoire, arrivent à pénétrer dans les lymphatiques de la capsule du nodule. Elles peuvent alors emprunter le système lymphatique pour arriver dans le derme superficiel.

Dans le tissu extra-nodulaire, les microfilaires rencontrées appartiennent vraisemblablement à plusieurs espèces d'onchocerques mais elles ne peuvent pas être identifiées. Les lésions induites sont superposables à celles observées chez l'homme onchocerquien, ainsi que chez les animaux parasités par d'autres filaires non nodulaires, mais à microfilaires dermiques, et appartenant ou non au genre Onchocerca. Les observations faites sur les bovins confirment et généralisent la notion que les microfilaires dermiques ne vivent pas librement dans le derme mais sont normalement lymphatiques (21, 22,23 ). Elles peuvent induire au niveau des capillaires lymphatiques des lésions de lymphangite. L'issue exceptionnelle de ces microfilaires hors des lymphatiques dermiques provoque une réaction inflammatoire dans le tissu conjonctif. Les onchocerques nodulaires bovines constituent un modèle intéressant pour l'onchocercose humaine.

\section{BIBLIOGRAPHIE}

1. BAIN (O.). Le genre. Onchocerca : hypothèses sur son évolution et clé dichotomique des espèces. Annls Parasit. hum. comp., 1981, 56 : 503-526.

2. BAIN (O.), DENKE (A.M.), AMEGEE (Y.), CHABAUD (A.G.). Les onchocerques des bovins au Togo, les microfilaires et leurs distributions. Annls Univ. Bénin, Togo, 1977, 3: 117-123.

3. BEVERIIGE (T.), KUMMEZROW (E.L), WILKINSON (P.). Observation on Onchocerca gibsoni and nodule. Development in naturally infected cattle in Australia. Trop. Med. Parasit., 1989, 31: 75-81.

4. BRUMPT (E.). Filaria volvulus.. Revue Méd. Hyg. trop., 1904, 1: 43-46.

5. BURCHARD (G.D.), BÜTTNER (D.W.), BIERTHER (M.). Electron microscopical studies on Onchocerciasis. III. The Onchocerca nodule. Tropenmed. Parasit., 1979, 30: 103-112.

6. BÜTTNER (D.W.), RACZ (P.). Macro and microfilariae in nodules from onchocerciasis patients in the Yemen Arab Republic. Tropenmed. Parasit, 1983, 34: 113-121.

7. CHODNIK (K.S.). Histopathology of aortic lesions in cattle infected with Onchocerca armillata (Filaridae). Annls trop. Med. Parasit, 1958, 5 (2): $145-148$

8. CI.ELAND (J.R.). Further investigations into the etiolngy of worm-nests in cattle, due to Onchocerca gibsoni.. Vet. J., London, 1914, 70: 551-555.

9. CONNOR (D.H.), NEAFIE (R.C.). Onchocerciasis. In : BINFORD (C.H.), CONNOR (D.H.). Pathology of tropical and extraordinary diseases. Washington, AFIP, 1976. p. 360-372. 
10. GILRUTH (J.A.), SWEET (G.). Onchocerca gibsoni : the cause of worm nodules in australian cattle. Sidney, Government Printer, 1911. p. 34.

11. HISSETTE (J.). Mémoire sur l'Onchocerca volvulus "Leuckart" et ses manifestations oculaires au Congo belge. Annls Soc. belge Med. trop., 1932, 12: 433-529.

12. JOHNSON (F.B.). Splendore-Hoeppli phenomenon. In : BINFORD (C.H.), CONNOR (D.H.). Pathology of tropical and extraordinary diseases. Washington, AFIP, 1976. p. 381-383.

13. LABADIE-LAGRAVE, DEGUY. Un cas de Filaria volvulus. Archs Parasit., 1899, 2: 451-460.

14. MARTINEZ-BAEZ (M.). Sur la structure histologique des nodules à Onchocerca volvulus et à O. caecutiens. Annls Parasit. hum. comp., 1935, 3: $207-230$.

15. MELLOR (P.S.). Studies on Onchocerca cervicalis Railliet and Henry, 1910. Part II. Pathology in the horse. J. Helminth., 1973, 47: 97-110.

16. MULLER (R.), HORSBURGH (R.C.R.). Bibliography of onchocerciasis (1841-1985). Oxon. C.A.B. International Institute of Parasitology, Reading, Eastern Press Ltd, 1987. 292 p.

17. PELLISSIER (A.). Le granulome lipidique dans la structure histologique des nodules sous-cutanés à Onchocerca volvulus. Bull. Soc. Path. exot., 1953, 46: 709-710.

18. RODHAIN (J.). Les filaires de l'Afrique tropicale: röle pathogène. Les réactions allergiques qu'elles provoquent et le diganostic de celles-ci. Bull. Soc. Path. exot., 1931, 24: 882-897.
19. SCHULZ-KEY (H.). The collagenase technique: how to isolate and examine adult Onchocerca volvulus for the evaluation of drug effects. Trop. Med. Parasit., 1988, 39 (suppl IV): 423-440.

20. SCHULZ-KEY (H.), ALBIEZ (E.J.). Worm burden of Onchocerca volvulus in a hyperendemic village of the Rain-Forest in West Africa. Tropenmed. Parasit., 1977, 28: 431-438.

21. VUONG-NGOC (P.), B AIN (O.), PETIT (G.), CHABAUD (A.G.). Etude comparative des lésions cutanées et oculaires du muridé Lemniscomys striatus, parasité par Monanema spp. et d'Atherurus africanus, parasité par Cercopithifilaria sp., avec celles de l'onclocerouse humaine. C.r. Acad. Sci., Paris, 1985, 301, sér III, (9): 433-435.

22. VUONG-NGOC (P.), BAIN (O.), PETIT (G.), CHABAUD (A.G.). Etude anatomo-pathologique des lésions cutanées et oculaires de rongeurs infestés par Monanema spp. Intérêt pour l'étude de l'onchocercose humaine. Annls Parasit. hum. comp.. 1986, 61 (3): 311-320.

23. VIUONG-NGOC (P.), RAIN (O.), CABARET (J.), PETIT (G.), PROD'HON (J.), RANQUE (Ph.), CHABAUD (A.G.). Forest and savanna onchocerciasis: comparative morphometric histopathology of skin lesions. Trop. Med. Parasit.. 1988, 39: 105-110.

24. VUONG (P.N.), WANJI (S.), SAKKA (S.), BAIN (O.). The murid filaria Monanema martini : A model for onchocerciasis. Part I. Description of lesions. Annls Parasit. hum. comp., 1991, 66 (3): 109-120.
VUONG (P.N.), WANJI (S.), PROD'HON (J.), BAIN (O.). Subcutanevus nudules and skin lesions caused by several Onchocerca spp. in African cattle. Revue Élev. Méd. vét. Pays trop., 1994, 47 (1) : 47-51

Subcutaneous nodules and skin biopsy specimens obtained from the umbilical area of 6 onchocercal Bos indicus were collected in Cameroon and subjected to a histological examination. The nodules containing Onchocerca ochengi and Onchocerca dukei showed the same structure as Onchocerca volvulus nodules in man ; they consisted of inflammatory pseudo-cysts often containing a female filaria. These pseudo-cysts were classified as "young", "active" and "old" depending on the inflammatory cellular component of their walls. The nodules were surrounded by a connective tissue capsule criss-crossed by vessels containing sections of microfilariae and morula in the vascular lumen. The skin, infected with microfilariae of these two species, of $O$. gutturosa and $O$. armillata, showed various dermatitis lesions with fibrosis, similar to those observed in patients with onchocerciasis. In most cases, inflammatory infiltrates surrounded the lymphatic capillaries along the blood vessels, giving rise to lymphangitis.The presence of microfilariae inside the granulomatous tissue demonstrates that microfilariae were deposited directly into the cystic lumen. Only the microfilariae which escaped the inflammatory reaction were able to penetrate the lymphatic vessels. They could then migrate into the lymphatic system and reach the superficial layer of the dermis. Bovine nodular onchocercomata represent an interesting model for human onchocerciasis.

Key words : Cattle - Onchocerciasis - Lesion - Skin disease - Lymphatic disease - Anatomo-pathology - Cameroon.
VUONG (P.N.), WANJI (S.), PROD'HON (J.), BAIN (O.). Nódulos subcutáneos y lesiones cutáneas causados por varios oncocercas en bovinos africanos. Revue Élev. Méd. vét. Pays trop., 1994, 47 (1) : 47-51

Se tomaron nódulos subcutáneos y de la piel umbilical de 6 Bos indicus teniendo oncocercas en Camerún, para el estudio anatomo-patologíco. Los nódulos con Onchocerca ochengi y $O$. dukei tienen la misma estructura que los con $O$. volvulus del hombre ; son seudoquistes inflamatorios encerrando a menudo una filaria hembra ; el componente celular de la pared del seudoquiste permite que se clasifiquen los nódulos en 3 tipos : nódulo "joven", nódulo "evolucionado" y nódulo "viejo" ; vasos cuya luz encerra a veces microfilarias y morulas limitan el nódulo. La piel, parasitada por las microfilarias de dichos dos oncocercas y por las de $O$. gutturosa y $O$. armillata, presenta varias lesiones de dermitis con esclerosis cicatrizal semejantes a las observadas en los enfermos teniendo la oncocercosis. Generalmente, los infiltrados inflamatorios limitan los capilares linfáticos extendiendose a lo largo de los vasos sanguíneos, realizando una linfangitis. Se pueden sobreponer todas estas lesiones a las observadas en los enfermos teniendo la oncocercosis. La presencia de microfilarias en el tejido de granulación bordeando el seudoquiste muestra que son aovadas en la luz del seudoquiste ; sólo llegan a penetrar en los linfáticos de la cápsula del nódulo las que no tienen una reacción inflamatoria ; entonces pueden tomar el sistema linfático para llegar en la dermis superficial. Los oncocercas nodulares bovinos constituyen un modelo interesante para la oncocercosis humana.

Palabras clave : Bovino - Oncocercosis - Lesión - Enfermedad de la piel - Enfermedad linfática - Anatomopatología - Camerún. 